\title{
Isolated Aphasic Status Epilepticus as a Manifestation Induced by Hyperglycemia Without Ketosis
}

\author{
Syuichi Tetsuka ${ }^{\mathrm{a}, \mathrm{c}}$, Nobuyuki Yasukawa ${ }^{\mathrm{b}}$, Asako Tagawa ${ }^{\mathrm{a}}$, Tomoko Ogawa ${ }^{\mathrm{a}}$, \\ Mieko Otsuka $^{\text {a }}$, Ritsuo Hashimoto ${ }^{\text {a }}$, Hiroyuki Kato ${ }^{a}$
}

\begin{abstract}
Diagnosis of aphasic status epilepticus (ASE) is very difficult, because the literature containing, to our knowledge, isolated non-ketotic hyperglycemia $(\mathrm{NKH})$-related ASE has reported only three cases. Although most cases are associated with organic brain lesions accompanying other clinical seizure activity, NKH-related ASE is rarer. We describe a rare case of NKH presenting initially as persistent and isolated ASE. Brain magnetic resonance imaging (MRI) did not reveal any focal lesion, but ictal electroencephalography (EEG) disclosed diffuse continuous theta to delta waves, intermingled with epileptiform discharges. Only correcting the hyperglycemia could not improve the language disorder completely, and the seizure was controlled only by the addition of phenytoin immediately. Patients with NKH may initially present with isolated ASE. Unlike stroke-related aphasia, accurate diagnosis is difficult if based solely on neurologic examination and brain neuroimaging. Use of EEG and blood sugar determination should be helpful in this special case. Prompt treatment including correcting the hyperglycemia would substantially improve the outcomes of NKH but might be insufficient. Additional prompt use of antiepileptic drugs might be potentially more effective.
\end{abstract}

Keywords: Aphasic status epilepticus; Non-ketotic hyperglycemia; Diagnosis; EEG; Insulin; Therapy; Antiepileptic drug

\section{Introduction}

Non-convulsive status epilepticus (NCSE) presents with minimal seizure activity clinically but is evident on electroenceph-

\footnotetext{
Manuscript accepted for publication August 29, 2016

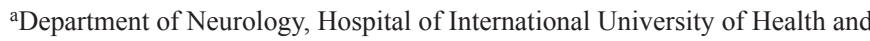
Welfare, 537-3, Iguchi, Nasushiobara, Tochigi 329-2763, Japan

bDepartment of Endocrinology \& Metabolism, Hospital of International University of Health and Welfare, 537-3, Iguchi, Nasushiobara, Tochigi 329-2763, Japan

${ }^{\mathrm{c} C o r r e s p o n d i n g ~ A u t h o r: ~ S y u i c h i ~ T e t s u k a, ~ D e p a r t m e n t ~ o f ~ N e u r o l o g y, ~ H o s p i t a l ~}$ of International University of Health and Welfare, 537-3, Iguchi, Nasushiobara, Tochigi 329-2763, Japan. Email: syuichi@jichi.ac.jp
}

doi: http://dx.doi.org/10.14740/jnr395w alography (EEG) [1]. Clinical features associated with NCSE in older people are associated with confusion including aphasia or interrupted speech. Isolated aphasic status epilepticus (ASE) is also a similar condition, albeit rare. Although aphasia during epileptic seizures with epileptic aphasia is not rare, isolated epileptic aphasia without other clinical seizure activity is rarer [2]. While hypoglycemia-induced seizures, which are usually generalized, have been extensively described in the literature, non-ketotic hyperglycemia (NKH) is associated with simple partial seizure, partial motor status, rarely complex partial seizure, or even with an isolated aphasic seizure without a localized lesion [3]. Without EEG, the diagnosis of epileptic aphasia is often missed, and the seizures can continue untreated for several more days, risking an aggravation of the ischemic deficit [4]. Isolated ASE without previous epilepsy, or cerebral lesions, is even rarer, especially due to NKH [2]. We present a rare case of NKH-related isolated ASE, successfully treated by phenytoin and good blood sugar control by insulin therapy.

\section{Case Report}

A 70-year-old right-handed woman experienced acute speech disturbance for about 7 days. Her speech was scanty and incomprehensible, and she could not understand spoken language when she awoke that morning. However, she was alert and could express her needs by gesturing. The course was persistent. The patient had been diagnosed with type 2 diabetes mellitus (DM) about 10 years ago. However, she had not received medical treatment for DM. Neurological examination revealed an alert and cooperative patient who had mixed aphasia with impaired fluency, repetition, comprehension, and naming. Muscle power was full, and no spontaneous muscle activity could be found. She would occasionally have spontaneous speech and could follow orders by gesturing.

When she was admitted to our hospital, initial laboratory data showed serum glucose $544 \mathrm{mg} / \mathrm{dL}$, osmolality 537 mOsm/L (normal level: 285 - 295), and HbA1c 15.2\%, without ketonuria or systemic acidosis. An initial diagnosis of NKH was made. Brain magnetic resonance imaging (MRI) showed mild cortical atrophy but no focal lesion. Cerebrospinal fluid (CSF) was within normal limits (CSF monocyte 2, protein 43 $\mathrm{mg} / \mathrm{dL}$ and $\mathrm{CSF} /$ blood glucose $185 \mathrm{mg} / \mathrm{dL}$ ). EEG on the next 


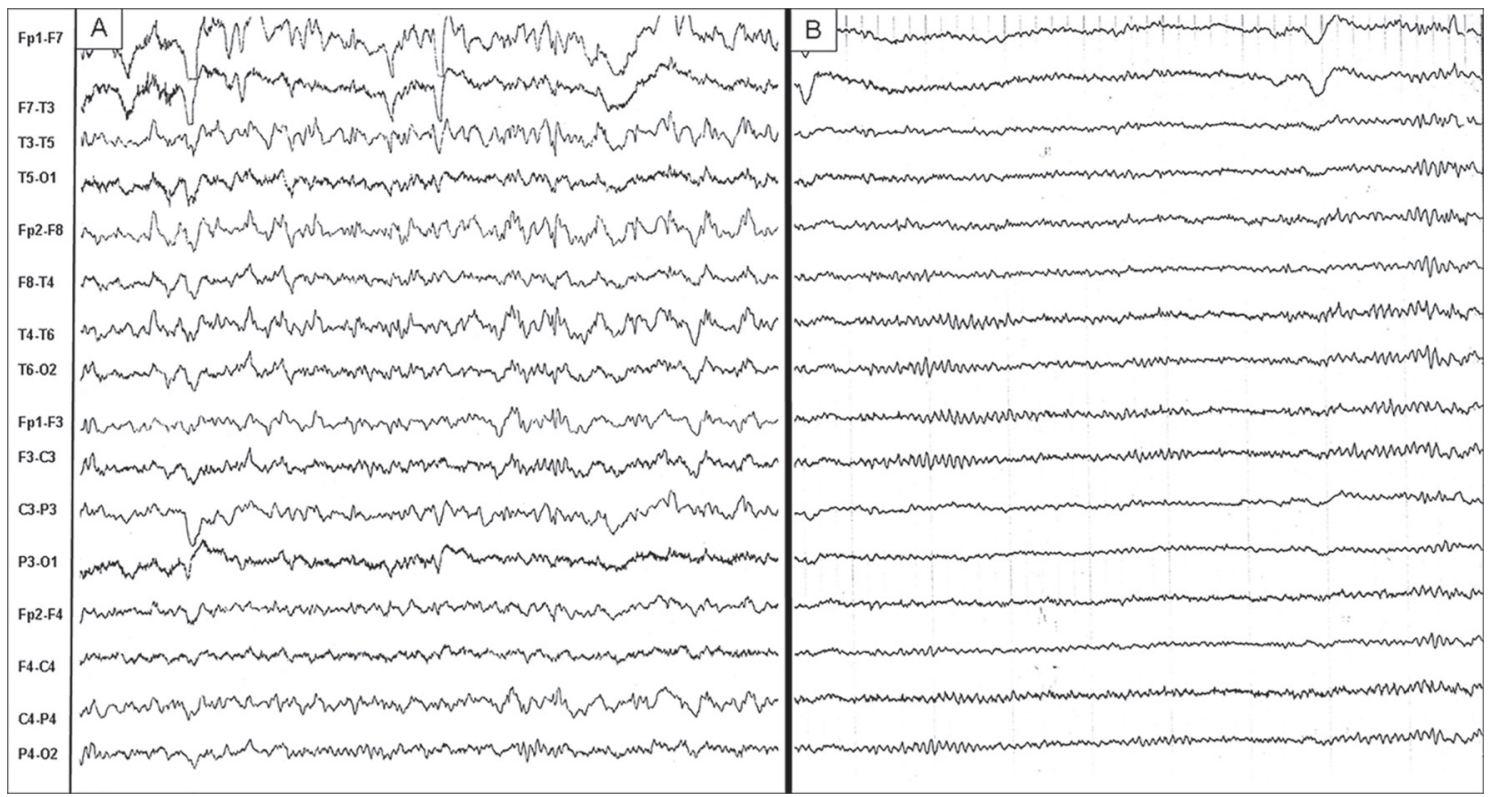

Figure 1. EEG showed diffuse continuous theta to delta waves intermingled with slightly left frontotemporal epileptiform discharges when aphasia persisted (A). EEG became normalized when aphasia subsided (B). EEG: electroencephalography.

day of admission showed diffuse continuous theta to delta waves intermingled with epileptiform discharges (Fig. 1A). Aphasic disorder, EEG epileptiform discharges, and a negative cranial MRI excluded cerebrovascular disease but included the possibility of "epileptic aphasia". A diagnosis of NKH-related isolated ASE was made.

The aphasia persisted, although blood sugar was controlled $(<200 \mathrm{mg} / \mathrm{dL}) 2$ days after admission. Fosphenytoin sodium hydrate intravenous injection was started 2 days after correction of hyperglycemia. Thereafter, phenytoin as an oral medicine was titrated to $200 \mathrm{mg} /$ day. Mixed aphasia gradually shifted to Broca's aphasia. Speech was restored and EEG normalized 2 days later (Fig. 1B). She said that she had no memory of about 1 week period until then. No aphasia or seizures were noted again, and blood sugar was controlled by insulin therapy. She left the hospital 2 weeks after admission. Upon discharge, she was not found to have minimal word-finding difficulties on detailed language testing. Thereafter, she did not develop a sudden recurrence or worsening of symptoms in the follow-up period, even after discontinuing phenytoin.

\section{Discussion}

Focal seizures induced by hyperglycemia were first reported in 1965 [5]. It was often encountered in clinical practice and was characterized by hyperglycemia, no keto-acidosis. Epileptic seizures occur in up to $25 \%$ of cases of $\mathrm{NKH}$, and in $50 \%$ of cases at the onset of diabetes [6]. The seizures were classically regarded as resistant to anticonvulsant treatment, and seizure control is associated with resolution of the hyperglycemia. Seizures related to $\mathrm{NKH}$ are usually partial seizures.

We presented a rare case of $\mathrm{NKH}$, initially presenting as isolated ASE. Although hyperglycemia was corrected, apha- sia was not controlled until the addition of phenytoin. Patients with ASE seem to have a symptomatic, often highly localized, cause for their seizures. Continuous EEG is necessary to make a definite diagnosis of ASE and monitor subsequent treatment [7]. EEG abnormalities of ASE include epileptiform discharges, periodic lateralized epileptiform discharges, and slow waves in the left frontal, temporal, and parietal cortex [7]. Although most patients with ASE have organic brain lesions, such as tumor, vascular abnormalities, stroke, or herpes simplex virus infection, NKH-related ASE is rare, especially if isolated without other clinical seizure features $[7,8]$. The clinical course of our patient fits ASE. However, the EEG findings of our patient were not typical unlike anything pointed out before, and showed diffuse epileptiform discharges, without focal abnormalities. The interictal abnormalities were most often found in the frontal [9] and more rarely in the temporal or occipital area, depending on the progression of simple partial seizures evolving to secondary ones.

On literature review, initial status epilepticus presenting with aphasia has been reported in multiple sclerosis [10], diabetes [11], NKH [3, 12], and AIDS-toxoplasmosis complex [13] or induced by the cephalosporin cefepime [14]. NKHrelated ASE seems to have its initial presentation as a mixed aphasia [12], similar to our case. This kind of aphasia is similar to that of patients with isolated aphasia, making it more difficult to establish an accurate diagnosis of NKH-related ASE, because of being very rare. To our knowledge, isolated NKHrelated ASE has been reported in only two cases without complications in the brain such as an old cerebral infraction $[3,12]$. Table 1 shows a summary of published cases of patients with isolated NKH-related ASE up to the present. In addition, cases of prolonged isolated ASE in patients without previous seizures or known cerebral lesions are rare as this case. Although isolated NKH-related ASE is very unusual at present, similar 
Table 1. Summary of Published Cases of Patients With Isolated NKH-Related ASE

\begin{tabular}{llllllll}
\hline $\begin{array}{l}\text { Age } \\
\text { (years) }\end{array}$ & Sex & $\begin{array}{l}\text { Serum glucose } \\
(\mathbf{m g} / \mathbf{d L})\end{array}$ & $\begin{array}{l}\text { Serum osmolality } \\
(\mathbf{m} \text { Osm/L) }\end{array}$ & $\begin{array}{l}\text { Abnormal region } \\
\text { in EEG }\end{array}$ & MRI findings & $\begin{array}{l}\text { Antiepileptic } \\
\text { drug }\end{array}$ & Prognosis \\
\hline 70 & F & 544 & 537 & Left frontotemporal & Normal & Phenytoin & $\begin{array}{l}\text { Drug free, no } \\
\text { recurrence }\end{array}$ \\
52 & M & 350 & 290 & Left temporal case & Normal & Carbamazepine & $\begin{array}{l}\text { Almost drug free, Pro et al, } \\
\text { no recurrence }\end{array}$ \\
78 & M & 381 & 297 & Left frontotemporal $[3]$ & $\begin{array}{l}\text { No focal } \\
\text { lesion }\end{array}$ & Carbamazepine & $\begin{array}{l}\text { Drug free, no } \\
\text { recurrence }\end{array}$ \\
\hline
\end{tabular}

EEG: electroencephalography; MRI: brain magnetic resonance imaging.

case reports may increase in the future, because neurologists had not recognized the value of that practical matter so far.

Seizures can be found in $25 \%$ of patients with NKH and may be the first manifestation in up to $50 \%$ of NKH patients with seizures [15]. Tiamkao et al [16] have proposed the following diagnostic criteria for NKH-related seizures: plasma glucose $>290 \mathrm{mg} / \mathrm{dL}$ and osmolarity $288 \mathrm{mOsm} / \mathrm{kg}$. Our patient had plasma glucose of $544 \mathrm{mg} / \mathrm{dL}$ and an osmolarity of $537 \mathrm{mOsm} / \mathrm{kg}$. Thus, our case fulfilled the criteria of Tiamkao et al.

Debates have continued about the mechanisms of seizures in NKH. Each mechanism, when considered alone, is unsatisfactory. The possible mechanisms proposed are hyperglycemia or hyperosmolarity, a low level of gamma aminobutyric acid, and focal ischemia [17]. Moreover, hyperglycemia may be a proconvulsant per se, even in the absence of organic brain lesions [18]. Decreased local brain blood flow, producing reversible focal ischemia, and decreased seizure threshold were also reported [19]. NKH-related seizures, generally considered as a type of symptomatic seizures, were noted to be usually resistant to anticonvulsant treatment but respond well to insulin therapy and rehydration [8]. However, only correction of hyperglycemia in our case failed to control NKH-related "epileptic aphasia", involving that it is more than a symptomatic seizure disorder unlike other metabolic disorders, or hypoglycemia-related generalized seizures. We supposed that NKH-related epileptic aphasia in our case is actually an epilepsy. Thus, the use of an anticonvulsant was necessary. Whether NKH-related epileptic aphasia is solely a symptomatic seizure disorder or is largely based on the degree of cerebral damage caused by NKH is not known. In addition, other neurological manifestations are reported in the literature, such lateral hemianopia, visual hallucinations, cortical blindness, recurrent giratory seizures, chorea or hemiballism or even scarce biballism, and of course, isolated ASE as in our case. The symptoms suggest that $\mathrm{NKH}$ cause damage to nerve cells in the brain. NKH itself can induce seizure disorders, and poor glycemic control can further aggravate epileptic seizures. The patients with diabetic hyperglycemia had more recurrent seizures and status epilepticus than those without, suggesting potential kindling during poor glycemic control [20]. Further detailed investigations on NKH-related epileptic aphasia are necessary.

Different types of seizures noted are motor seizures, usually focal, rarely generalized tonic-clonic seizures. Seizures associated with $\mathrm{NKH}$ are often recurrent, and partial status epilepticus has been observed. The prognosis of seizures and movement disorders related to $\mathrm{NKH}$ is dependent on treatment involving correction of the hyperglycemia with insulin treatment and rehydration [21], and it would be indeed the same in all patients as the present case with isolated ASE.

In summary, patients with NKH may initially present with persistent and isolated ASE. Unlike stroke-related aphasia, NKH-related epileptic aphasia is difficult to diagnose accurately if based solely on neurologic examination and cranial neuroimaging. The use of EEG and blood sugar examination should be helpful in all patients presenting with acute aphasia. Prompt treatment including correcting the hyperglycemia would substantially improve the outcomes of NKH but might be insufficient. Additional prompt use of antiepileptic drugs might be potentially more effective.

\section{Acknowledgments}

The authors are grateful for the patient in accepting to include her clinical and radiological information for publication.

\section{Consent}

Written informed consent was obtained from the patient for publication of this case report and any accompanying images.

\section{Conflicts of Interest}

The authors declare that they have no conflicts of interest.

\section{References}

1. Woodford HJ, George J, Jackson M. Non-convulsive status epilepticus: a practical approach to diagnosis in confused older people. Postgrad Med J. 2015;91(1081):655661.

2. Flugel D, Kim OC, Felbecker A, Tettenborn B. De novo status epilepticus with isolated aphasia. Epilepsy Behav. 2015;49:198-202.

3. Pro S, Randi F, Pulitano P, Vicenzini E, Mecarelli O. Nonconvulsive status epilepticus characterised exclusively by 
a language disorder induced by non-ketotic hyperglycaemia. Epileptic Disord. 2011;13(2):193-196.

4. Lynch MW, Rutecki PA, Sutula TP. The effects of seizures on the brain. Curr Opin Neurol. 1996;9(2):97-102.

5. Maccario M, Messis CP, Vastola EF. Focal Seizures as a Manifestation of Hyperglycemia without Ketoacidosis. A Report of Seven Cases with Review of the Literature. Neurology. 1965;15:195-206.

6. Chung SJ, Lee JH, Lee SA, No YJ, Im JH, Lee MC. Cooccurrence of seizure and chorea in a patient with nonketotic hyperglycemia. Eur Neurol. 2005;54(4):230-232.

7. Ericson EJ, Gerard EE, Macken MP, Schuele SU. Aphasic status epilepticus: electroclinical correlation. Epilepsia. 2011;52(8):1452-1458.

8. Younes S, Cherif Y, Aissi M, Alaya W, Berriche O, Boughammoura A, Frih-Ayed M, et al. Seizures and movement disorders induced by hyperglycemia without ketosis in elderly. Iran J Neurol. 2014;13(3):172-176.

9. Kaplan PW, Rossetti AO, Kaplan EH, Wieser HG. Proposition: limbic encephalitis may represent limbic status epilepticus. A review of clinical and EEG characteristics. Epilepsy Behav. 2012;24(1):1-6.

10. Trinka E, Unterberger I, Spiegel M, Niedermuller U, Benke T, Berger T, Bauer G. De novo aphasic status epilepticus as presenting symptom of multiple sclerosis. J Neurol. 2002;249(6):782-783.

11. Dinner DS, Lueders H, Lederman R, Gretter TE. Aphasic status epilepticus: a case report. Neurology. 1981;31(7):888-891.

12. Huang LC, Ruge D, Tsai CL, Wu MN, Hsu CY, Lai CL, Liou LM. Isolated aphasic status epilepticus as initial presentation of nonketotic hyperglycemia. Clin EEG
Neurosci. 2014;45(2):126-128.

13. Ozkaya G, Kurne A, Unal S, Oguz KK, Karabudak R, Saygi S. Aphasic status epilepticus with periodic lateralized epileptiform discharges in a bilingual patient as a presenting sign of "AIDS-toxoplasmosis complex". Epilepsy Behav. 2006;9(1):193-196.

14. Kwon J, Choi JY, Bae EK. Cefepime-induced Aphasic Status Epilepticus Mimicking Acute Stroke. J Epilepsy Res. 2014;4(2):85-87.

15. Lammouchi T, Zoghlami F, Ben Slamia L, Grira M, Harzallah MS, Benammou S. [Epileptic seizures in nonketotic hyperglycemia]. Neurophysiol Clin. 2004;34(34):183-187.

16. Tiamkao S, Pratipanawatr T, Nitinavakarn B, Chotmongkol V, Jitpimolmard S. Seizures in nonketotic hyperglycaemia. Seizure. 2003;12(6):409-410.

17. Brick JF, Gutrecht JA, Ringel RA. Reflex epilepsy and nonketotic hyperglycemia in the elderly: a specific neuroendocrine syndrome. Neurology. 1989;39(3):394-399.

18. Schwechter EM, Veliskova J, Velisek L. Correlation between extracellular glucose and seizure susceptibility in adult rats. Ann Neurol. 2003;53(1):91-101.

19. Suarez-Moro R, Salas-Puig J, Amorin M, Roiz C, Lahoz C. SPECT findings in reflex seizures induced by movement in non-ketotic hyperglycemia. Epileptic Disord. 1999;1(3):199-201.

20. Huang CW, Tsai JJ, Ou HY, Wang ST, Cheng JT, Wu SN, Huang CC. Diabetic hyperglycemia is associated with the severity of epileptic seizures in adults. Epilepsy Res. 2008;79(1):71-77.

21. Scherer C. [Seizures and non-ketotic hyperglycemia]. Presse Med. 2005;34(15):1084-1086. 